\title{
The evolution of groups and clusters
}

\section{S. Gottlöber}

Astrophysical Institute Potsdam, An der Sternwarte 16, 14482 Potsdam, Germany

\author{
A. Klypin and. A. V. Kravtsov
}

Astronomy Department, NMSU, Dept.4500, Las Cruces, NM 88003-0001, USA

\section{Turchaninov}

Keldysh Institute for Applied Mathematics, Miusskaja Ploscad 4, 125047 Moscow, Russia

\begin{abstract}
Using high resolution $N$-body simulations we study the formation and evolution of clusters and groups in a $\Lambda \mathrm{CDM}$ cosmological model. Groups of galaxies already form before $z=4$. Merging of groups and accretion leads to cluster formation at $z \lesssim 2$. Some of the groups merge into large isolated halos.
\end{abstract}

\section{Introduction}

Cosmological scenarios with cold dark matter alone cannot explain the structure formation both on small and very large scales. Scenarios with a non-zero cosmological constant $\Lambda$ have proved to be very successful in describing most of the observational data at both low and high redshifts. Moreover, from a recent analysis of 42 high-redshift supernovae, Perlmutter et al. (1999) found direct evidence for $\Omega_{\Lambda}=0.72$ within a flat cosmology.

It is generally believed that the dark matter component of galaxies, the dark matter halos, plays an important role in the formation of galaxies. Properties and evolution of the halos depend on the environment (Avila-Reese et al. 1999, Gottlöber et al. 1999a, b) which implies, in turn, that properties of galaxies can be expected to depend on cosmological environment too. Many of the dark matter halos are much more extended than galaxies and contain several subhalos. Here we present results of a study of the formation and evolution of these large halos which host groups and clusters of galaxies.

\section{Dark matter halos in the simulation}

We simulate evolution of $256^{3}$ cold dark matter particles in a $\Lambda$ CDM model $\left(\Omega_{M}=1-\Omega_{\Lambda}=0.3 ; \sigma_{8}=1.0 ; H_{0}=70 \mathrm{~km} \mathrm{~s}^{-1} \mathrm{Mpc}^{-1}\right)$. As a compromise we have chosen a simulation box of $60 \mathrm{~h}^{-1} \mathrm{Mpc}$ in order to study the statistical 


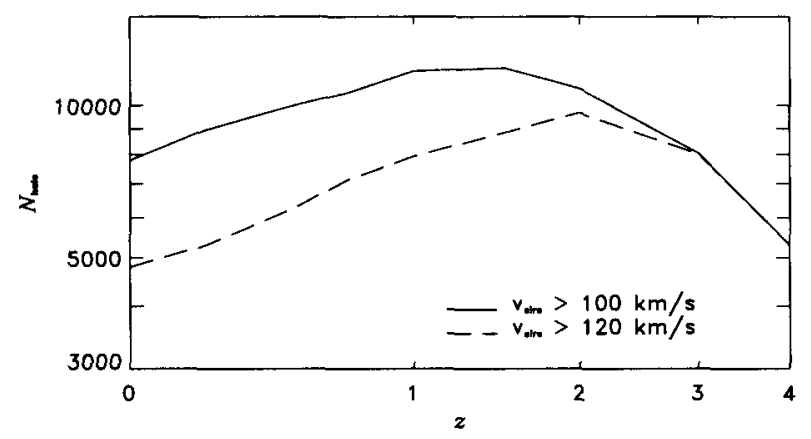

Figure 1. The number of halos with more than 50 bound particles depending on redshift for $v_{\text {circ }}>100 \mathrm{~km} \mathrm{~s}^{-1}$ (solid line) and $v_{\text {circ }}>120$ $\mathrm{km} \mathrm{s}^{-1}$ (dashed line).

properties of halos in a cosmological environment and to have also a sufficient mass resolution (particle mass of $\left.1.1 \times 10^{9} h^{-1} M_{\odot}\right)$. The simulations were done using the Adaptive Refinement Tree (ART) code (Kravtsov, Klypin \& Khokhlov 1997). The code used a $512^{3}$ homogeneous grid on the lowest level of resolution and six levels of refinement, each successive refinement level doubling the resolution. The sixth refinement level corresponds to a formal dynamical range of 32,000 in high density regions. Thus we can reach in a $60 h^{-1} \mathrm{Mpc}$ box a force resolution of $\approx 2 h^{-1} \mathrm{kpc}$.

Identification of halos in dense environments and reconstruction of their evolution is a challenge. In order to find halos in the simulation we have developed two algorithms, which we called the hierarchical friends-of-friends (HFOF) and the bound density maxima (BDM) algorithms (Klypin et al. 1999). These algorithms work on a snapshot of the particle distribution. They are able to identify "halos within halos", i.e. stable gravitationally self-bound halos which move inside a larger region of virial overdensity. Therefore, we can find dark matter halos which correspond to galaxies inside of groups or clusters but also small satellites bound to a larger galaxy (like the LMC and the Milky Way or the M51 system). The algorithm identifies halos at different redshifts. In a second step we establish the "ancestor-descendant" relationships for all halos at all times (Gottlöber et al. 1999a, b).

The total mass of a halo depends on its assumed radius. However, for satellite halos within larger bound systems the notion of radius becomes somewhat arbitrary. The formal virial radius of a satellite halo is simply equal to the virial radius of its host halo. We have chosen to define the outer tidal radius of the satellite halos as the radius at which their density profile starts to flatten. We try to avoid the problem of mass determination by assigning not only a mass to a halo, but finding also its maximum "circular velocity" $v_{\text {circ }}=\sqrt{G M / R}$. This is the quantity which is more meaningful observationally. Numerically, $v_{c i r c}$ can be measured more easily and more accurately than mass. 
In order to define a complete halo sample that is not affected by the numerical details of the halo finding procedure we have constructed the differential velocity functions at $z=0$ for different mass thresholds and maximum radii. We found that the halo samples do not depend on the numerical parameters of the halo finder for halos with $v_{\text {circ }} \gtrsim 100 \mathrm{~km} \mathrm{~s}^{-1}$ (Gottlöber et al. 1999a). Here we want to study samples of halos at redshifts $z \leq 4$ with circular velocities $v_{\text {circ }}>100 \mathrm{~km} \mathrm{~s}^{-1}$ and, for comparison, samples of more massive halos with circular velocities $v_{\text {circ }}>120 \mathrm{~km} \mathrm{~s}^{-1}$. Moreover, we include only halos which consist of at least 50 bound particles. This reduces the possible detection of fake halos in the simulation, in particular the detection of very small fake satellite halos. At redshift $z=0$ we have detected 7786 halos with $v_{\text {circ }}>100 \mathrm{~km} \mathrm{~s}^{-1}$ in our simulation, which corresponds to a number density of $0.036 h^{3} \mathrm{Mpc}^{-3}$. 4787 of these halos have circular velocities $v_{\text {circ }}>120 \mathrm{~km} \mathrm{~s}^{-1}\left(0.022 h^{3} \mathrm{Mpc}^{-3}\right)$. These densities roughly agree with the number density of galaxies with magnitudes $M_{r} \lesssim-16.3$ and $M_{r} \lesssim-18.4$ estimated by the luminosity function of the Las Campanas redshift survey (Lin et al. 1996) which we have extended to -16.3 .

The redshift evolution of the total number of halos in the box is shown in Fig. 1. At $z=4$ we found the same number of halos independent of the threshold of the circular velocity. This means that we have reached here the resolution limit of the simulation. In fact, we had excluded low mass halos with less than 50 particles.

\section{Environment of Halos}

To find the cosmological environment of each of these halos at $z \leq 4$ we run a friend-of-friend analysis over the dark matter particles with a linking length of 0.2 times the mean interparticle distance. By this procedure we find clusters of dark matter particles with an overdensity of about 200. At $z=0$ the virial overdensity in the $\Lambda \mathrm{CDM}$ model under consideration is about 330 which corresponds to a linking length of about 0.17 . With increasing $z$ the virial overdensity decreases and reaches 200 at $z=1$. Here we have used the same linking length for all $z$. Therefore, the objects which we find at $z<1$ are slightly larger than the objects at virial overdensity. We have increased the linking length at $z=0$ because we found in the vicinity of galaxy clusters halos which had already interacted with the cluster but were in the moment of detection just outside the region of virial overdensity.

In a second step we find for each of the halos at all $z$ the cluster of dark matter particles to which the halo belongs. We call the halo a cluster galaxy if the the halo belongs to a particle cluster with a total mass larger than $10^{14} h^{-1} M_{\odot}$. We call it an isolated galaxy if only one halo belongs to the object at overdensity 200 which we found by the friends-of-friends algorithm. This definition ensures that the "isolated galaxies" really do not interact with other galaxies. We found a substantial number (about $10 \%$ ) of doublets. In some cases we found pairs of halos with approximately the same mass but in most of the cases these doublets consist of one big halo with a small bound satellite which is within or partly overlaps the larger halo. On one hand, there could be more small satellites which we do not detect due to the limited mass resolution so that the doublet is in fact a small group. On the other hand, there could be really only one small 


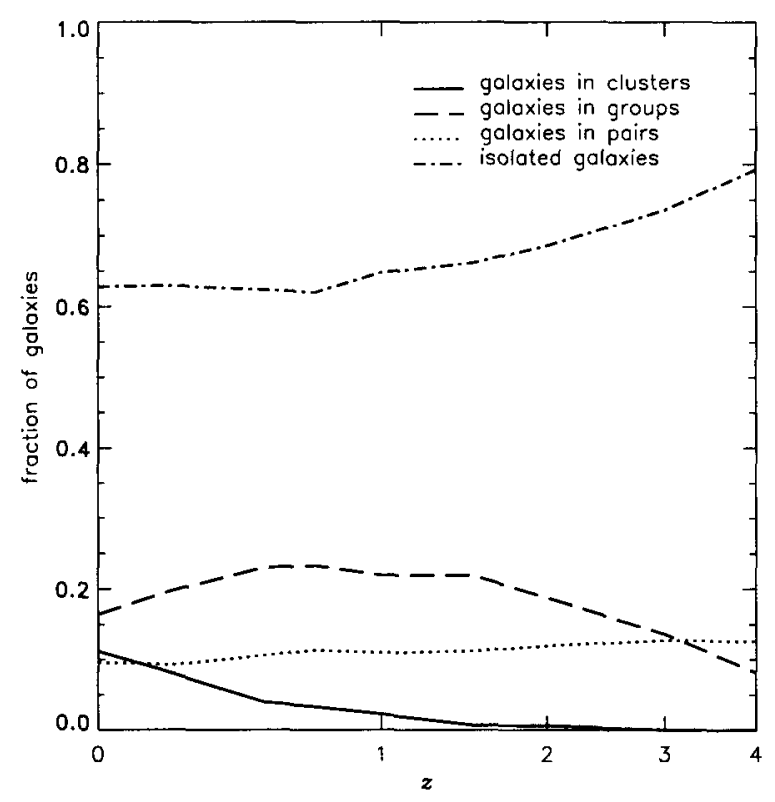

Figure 2. The evolution of the fraction of galaxies in clusters, groups and pairs and of isolated galaxies for dark matter halos with $v_{\text {circ }}>100$ $\mathrm{km} \mathrm{s}^{-1}$.

satellite so that the object could be better handled as an isolated galaxy. In order to avoid difficult decisions, we have handled the galaxy pairs separately having in mind that some of them could belong to isolated galaxies whereas other are seeds of a small group. The remaining halos are called group galaxies.

The 7786 halos with $v_{\text {circ }}>100 \mathrm{~km} \mathrm{~s}^{-1}$ are distributed over 18 clusters, 252 groups and 373 pairs, 4892 halos are isolated.

\section{The Evolution of Halos}

The total number of halos with $v_{\text {circ }}>100 \mathrm{~km} \mathrm{~s}^{-1}$ increases from 5290 at $z=4$ to the maximum value of 11847 at redshift $z=1.5$ and then decreased to 7786 at redshift $z=0$ (see Fig. 1). Assuming that each of these halos would contain a galaxy we compute the evolution of the fraction of isolated "galaxies" and "galaxies" in clusters, groups, and pairs.

The first object of virial mass $>10^{14} h^{-1} M_{\odot}$ forms between $z=2.5$ and $z=2$. At $z=2$ this cluster of $1.3 \times 10^{14} h^{-1} M_{\odot}$ already contains 68 satellite halos. The most massive central halo of this cluster has a circular velocity of $760 \mathrm{~km} \mathrm{~s}^{-1}$. The number of cluster galaxies increases up to $z=0$, where 868 galaxies have been detected in clusters.

At all analyzed time epochs in the simulation, we found approximately $10 \%$ of pairs. We have detected 113 groups already at $z=4$. These groups contain 


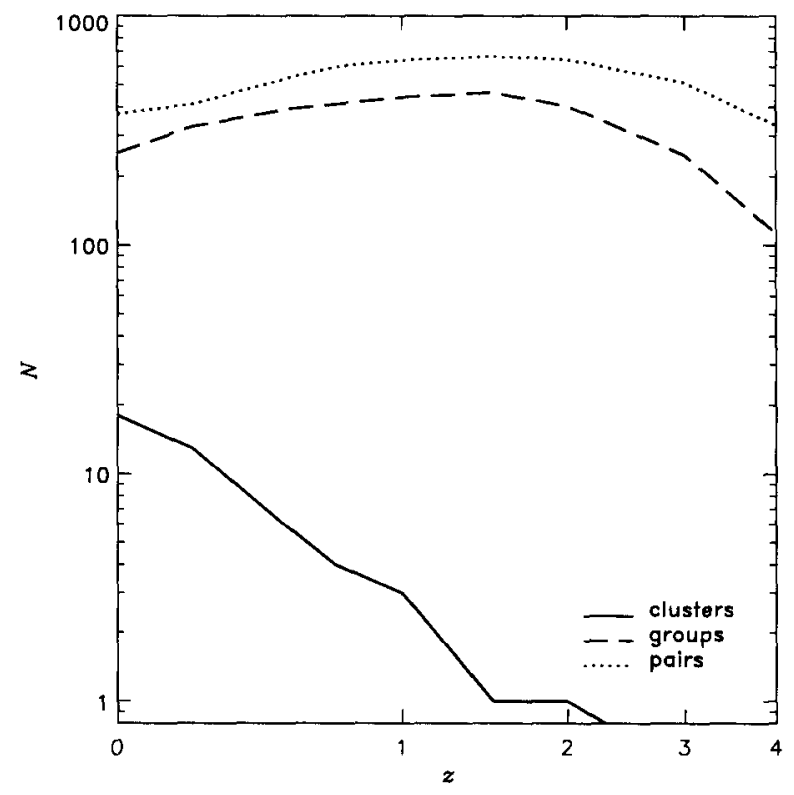

Figure 3. The evolution of the number of clusters, groups and pairs for dark matter halos with $v_{\text {circ }}>100 \mathrm{~km} \mathrm{~s}^{-1}$.

430 galaxies. The number of group galaxies increases very rapidly and reaches a maximum of 2607 at $z=1.5$. Afterwards it decreases to 1280 at $z=0$. Finally, also the number of isolated galaxies increases from $z=4$ (4194) until $z=1.5$ (7854) and then decreases until $z=0$ (4892).

In Fig. 2 we show the time evolution of the fraction of isolated galaxies and galaxies in clusters, groups, and pairs for dark matter halos with $v_{\text {circ }}>100$ $\mathrm{km} \mathrm{s}^{-1}$. The sample of more massive halos $\left(v_{\text {circ }}>120 \mathrm{~km} \mathrm{~s}^{-1}\right)$ shows essentially the same behavior, but the total number of halos is smaller.

Now let us discuss the evolution of clusters and groups. To this end, we find similar to the chain of progenitors of each halo the progenitors of the clusters and groups. The first cluster has been formed before $z=2$. Due to accretion and merging of groups the number of clusters increases to 18 at $z=0$ (Fig. 3). At $z=4$ we found 113 groups and 333 pairs in our simulation. It is remarkable that these numbers do not depend on the chosen threshold of the circular velocity. Both the number of groups and pairs increases up to $z=1.5$ where we have 465 (363) groups and $666(567)$ pairs for $v_{\text {circ }}>100 \mathrm{~km} \mathrm{~s}^{-1}\left(v_{\text {circ }}>120 \mathrm{~km} \mathrm{~s}^{-1}\right)$. At $z=0,252(152)$ groups and $373(258)$ pairs remain. The total number of groups and pairs reduces with increasing threshold of the circular velocity. In fact, if we take into account only more massive objects some of the pairs would be identified as isolated galaxies whereas some of the groups would be identified as pairs or isolated galaxies. However, as we already mentioned, the overall fraction of galaxies in groups and pairs is rather insensitive to this threshold. 
From Fig. 2, we see that the fraction of galaxies in groups decreases from about $22 \%$ at $z=0.7$ to about $16 \%$ at $z=0$. At the same time, the fraction of isolated halos slightly increases. The decreasing number of groups is mostly due to the fact that groups merge into more massive objects. However, we also found a small fraction of groups which merge into isolated halos. In the simulation we found 20 halos with masses larger than $10^{12} h^{-1} M_{\odot}$ the progenitor of which at $z=1$ was a group with 4 to seven members. Such a merged group could appear today as an isolated elliptical with a group-like X-ray halo (Mulchaey \& Zabludoff 1999; Vikhlinin et al. 1999).

\section{Conclusions}

The general trend during evolution of clustering is the formation of small bound systems of halos at $z \gtrsim 1.5$. After $z=1.5$ these systems tend to merge and to increase mass by accretion so that large galaxy clusters grow in the simulation. The total number of small bound systems and the total number of galaxies in these small systems rapidly decreases after $z=1.5$. The fraction of isolated galaxies remains approximately constant after $z=1$, whereas the fraction of galaxies in groups decreases. Some of the groups merge after $z=1$ to form large isolated galaxies.

Acknowledgments. This work was funded by the NSF and NASA grants to NMSU. SG acknowledges support from Deutsche Akademie der Naturforscher Leopoldina with means of the Bundesministerium für Bildung und Forschung grant LPD 1996. We acknowledge support by NATO grant CRG 972148.

\section{References}

Avila-Reese, V., Firmani, C., Klypin, A., Kravtsov, A. V., 1999, MNRAS, accepted, astro-ph/9906260

Gottlöber, S., Klypin, A., Kravtsov, A. V. 1999a, ASP Conference Series Vol. 176, Observational Cosmology: The Development of Galaxy Systems, Eds.: G. Giuricin, M. Mezetti, P. Salucci, p. 418.

Gottlöber, S., Klypin, A., Kravtsov, A. V., 1999b, astro-ph/9909012

Klypin, A., Gottlöber, S., Kravtsov, A. V., Khokhlov, A. M. 1999, ApJ, 516, 530

Kravtsov, A. V., Klypin, A., Khokhlov, A. M., 1997, ApJS, 111, 73

Lin, H., Kirshner, R. P., Shectman, S. A. et al., 1996, ApJ, 464, 60

Mulchaey, J. S., Zabludoff, A. I., 1999, ApJ, 514, 133

Perlmutter, S., Aldering, G., Goldhaber, G. et al., 1999, ApJ, 517, 565

Vikhlinin, A., McNamara, B. R., Hornstrup, A. et al., 1999, ApJL 520, L1 